REVISTA ECONOMÍA

Vol. 69, N. ${ }^{\circ}$ Io9 (mayo), I49-I59

\title{
ASOCIATIVIDAD Y VENTAS: UNA MIRADA DE LAS UNIDADES DE ECONOMÍA POPULAR Y SOLIDARIA EN EL ECUADOR
}

\author{
SEBASTIÁN BURGOS DÁVILA', GABRIELA FALCONÍ GUAMÁN² \\ Centro de Investigación Cuantitativa ECONÓMICA-CIC ${ }^{1}$, Investigadora independiente ${ }^{2}$
}

Recepción manuscrito: 6 de enero de 2017

Aceptación versión final: 15 de abril de 2017

\begin{abstract}
RESUMEN La economía popular y solidaria representa un nuevo esquema en el análisis de la firma en la cual los mecanismos de asociatividad juegan un importante rol dentro en la maximización de beneficios económicos. Un esquema asociativo permite a las unidades de economía popular y solidaria obtener mejores condiciones para su desarrollo. Con base a datos del Censo Nacional Económico de 2010 se encontró que la asociatividad tiene un efecto positivo en las ventas de los agentes de esta forma de producción influyendo en un incremento del diez por ciento en las ventas con relación a los agentes que no poseen ninguna relación de asociatividad.
\end{abstract}

PALABRAS CLAVE Economía popular y solidaria, teoría de la firma, asociatividad, ingresos.

ABSTRACT The popular and solidarity economy represents a new scheme in the analysis of the firm in which the mechanisms of associativity play an important role inside in the maximization of economic benefits. An associative scheme allows popular and solidarity economy units to obtain better conditions for their development. Based on data from the 2010 National Economic Census, it was found that associativity has a positive effect on the sales of the agents of this form of production influencing a ten percent increase in sales in relation to the agents that have no relation of associativity.

KEYWORDS Popular and solidarity economy, firm theory, associativity, income.

JEL CODES $\mathrm{O}_{12}, \mathrm{O}_{43}, \mathrm{O}_{54}, \mathrm{P}_{52}$.

\section{INTRODUCCIÓN}

La teoría económica clásica impone que el comportamiento de la firma se concentra en la maximización de beneficios considerando aspectos como el empleo como un simple factor de producción sin tomar en cuenta aspecto de redistribución. La economía popular y solidaria (EPS) se presenta como un nuevo paradigma en la teoría económica en donde la maximización de beneficios no es la finalidad de proceso productivo, sino un mecanismo de redistribución entre los individuos que conforma este esquema económico. Carranza (2013) expone una nueva visión de la economía popular y solidaria en la cual deja de un lado la perspectiva 
del agente que maximiza beneficios económicos bajo comportamientos egoístas y propone un nuevo agente racional que busca prácticas de reciprocidad y altruismo para lograr beneficios dentro de un mercado.

Siguiendo esta propuesta, la asociatividad es uno de los principios que deben regir a la economía popular y solidaria considerando el sentido altruista y de búsqueda del bienestar colectivo. En este sentido, es necesario elaborar un ejercicio que permita combinar el accionar de la EPs dentro de una lógica de mercado considerando dos aristas fundamentales: i) la economía popular y solidaria se concentra en una mejor redistribución de los beneficios económicos; y ii) los agentes de este subconjunto de la economía se enfrentan a una lógica comercial y de mercado que busca maximizar dichos beneficios. En este aspecto, se debe comprender que los agentes de la economía popular y solidaria tienen como incentivo la búsqueda del lucro dentro del esquema de mercado, pero la lógica de distribución de atributos cambia con relación al agente de producción clásico.

La asociatividad tienen efectos positivos dentro del funcionamiento de una firma; considerando que este fenómeno influye tanto a los agentes de la EPs como a los de la economía de mercado, es necesaria la cuantificación de este efecto sobre variables como las ventas de la firma. Con base en datos correspondientes al Censo Nacional Económico de 2010, se cuantifica la magnitud del efecto causal de la asociatividad sobre las ventas de las unidades de la economía popular y solidaria en el Ecuador. Para dicha finalidad se emplea la metodología de variables instrumentales que permiten cuantificar los efectos causales de un fenómeno sobre una variable en específico.

Este artículo se desarrolla de la siguiente manera: una sección primera de revisión de la literatura en relación a los beneficios que tiene la asociatividad en el desempeño de la firma, la segunda explica la metodología de variables instrumentales, la sección tercera detalla los resultados descriptivos y de las estimaciones realizadas; finalmente, la cuarta sección detalla las conclusiones de la presente investigación.

\section{ASOCIATIVIDAD E IMPACTOS EN EL COMPORTAMIENTO DE LA FIRMA}

La economía popular y solidaria es un paradigma económico que ha tenido un asidero conceptual muy fuerte en economías en desarrollo. Esta nueva forma de economía, basada en la maximización de la redistribución de las ganancias fruto de la actividad económica, no posee estudios en los que se cuantifique los beneficios que tiene sus principales características tales como la asociatividad. En este sentido se aprecia que los estudios relacionados a la asociatividad y sus efectos en variables cuantitativas se fundamentan más en lógicas de mercado y en relación a los tamaños que tienen las unidades de producción.

Estudios realizados por Aragón y Rubio (2005), Aragón y Sánchez (2005) Gálvez et al. (2012) demuestra que, desde una lógica de mercado, la asociatividad permite un mejor desempeño de las empresas pequeñas, ya que este factor permite que los niveles de riesgo se redistribuyan en los asociados, así como permiten mejorar la capacidad instalada y aumento de la producción. Evidentemente, los estudios mencionados presentan una lógica de empresa y no toman en cuenta aspectos propios de la economía popular y solidaria tales como la redistribución de ganancias y maximización del empleo. 
La asociatividad se encuentra muy ligada al crecimiento y desarrollo de la firma; desde un punto de vista microeconómico de la teoría de la firma, los esquemas asociativos provocan que la firma permita tener una restricción presupuestaria mayor, tasas marginales de sustitución técnica más grandes y costos marginales menores con lo que se garantiza una mejora en la función de producción, así como un mayor margen de beneficios (Varian, 2002). Desde el esquema de acción en las que las unidades de EPs se desarrollan, se puede determinar que este tipo de firmas se encuentran bajo un régimen de competencia perfecta, es decir, bajo una lógica de tomadores de precios y una ampliación de su frontera de posibilidades de producción gracias a la asociatividad se tiene un impacto positivo en los resultados de aprovechamiento de los beneficios producto de la asociación de agentes productivos.

Dentro de los casos de estudio, García et al. (2014) demuestran que las mipymes asociadas en Colombia tienen un incremento de ventas de aproximadamente $14 \%$. Si bien el estudio en mención utiliza solamente herramientas de carácter descriptivo, se presenta un indicio de las bondades que tiene la asociatividad en los resultados operativos de las firmas. ${ }^{1}$ Bajo una óptica de administración de empresas, la asociatividad y los esquemas de cooperación permiten una construcción de alianzas que permiten aprovechar de mejor manera los recursos que poseen cada una de las firmas y crear economías a escala que otorgan a la firma beneficios en cuanto a la reducción de su estructura de costos y mejoras en el uso de los insumos productivos y mejora los esquemas de competitividad (Ojeda, 2009).

Una de las ventajas que tiene la asociatividad es que no solo se puede dar entre unidades productivas del mismo sector industrial, al contrario, puede darse en toda la cadena de producción que tiene la firma (Colmenarez y Delgado, 2003). En este sentido, las acciones asociativas pueden dar un impulso hacia un crecimiento endógeno ya que toda la cadena de consumo intermedio puede ser suplida entre agentes que han llegado a este tipo de acuerdos. Estudios como los realizados por Salcedo Baca (2005) y Schejtman (1986) en cadenas productivas de alimentos, demuestran que la sinergia positiva entre asociatividad y cadenas de producción. ${ }^{2}$

En síntesis, cualquier acción que la unidad productiva emprenda en torno a la asociatividad tiene resultados positivos en el campo de la producción y del aprovechamiento de los beneficios propios de la competencia perfecta. Si bien este último enunciado tiene una perspectiva alejada de la EPS, es necesario considerar el accionar de este tipo de unidades dentro de un mercado de competencia perfecta en el que se desarrollan. Rescatando los beneficios de mercado que otorga la asociatividad, y sumando a las características que la EPs provee, se puede determinar la importancia y el impulso que se deben dar a este tipo de esquemas dentro de las unidades de producción de la economía popular y solidaria.

\section{METODOLOGÍA}

Las estimaciones por regresión de mínimos cuadrados ordinarios (MCO) deben cumplir con diferentes supuestos. Los supuestos de heterocedasticidad y no linealidad de los errores se corrigen fácilmente realizando correcciones a los errores estándar; sin embargo, el supuesto de exogeneidad con el cual se establece causalidad del fenómeno debe ser corregido con una variable instrumental (vI). En ciencias sociales, muchas de las variables analizadas o utilizadas 
no son exógenas - no son aleatorias-, es decir, dependen de otro tipo de variables en su determinación.

La metodología de variables instrumentales es una herramienta ampliamente utilizada en la corrección de endogeneidad de las variables; existe una serie de aplicaciones de esta metodología de los que sobresalen Andrabi et al. (2006), Angrist (1990), Blundell y Costa (2008), Glewwe y Jacoby (1995), Heckman (1997), Imbens y Angrist (1994), Moffit (2008), entre otros. Se recomienda que la variable instrumental no se encuentre asociada a fenómenos sociales; la literatura establece que variables relacionadas a fenómenos naturales o distribución geográfica son consideradas como los mejores instrumentos (Ravallion y Wodon, 200o; Semykina y Wooldridge, 2005). Tomando en cuenta la correlación del error estándar con la variable que provoca endogeneidad y el aislamiento al error que efectúa la variable instrumental, se puede concluir que esta metodología permite eliminar los efectos endógenos de la variable analizada dentro del error estándar (Wooldridge, 2009). El modelo se lo expresa de la siguiente manera:

$\mathrm{Y}_{\mathrm{i}}=\alpha \mathrm{X}_{\mathrm{i}}+\beta \mathrm{S}_{\mathrm{i}}+\varepsilon_{\mathrm{i}}(1)$

En donde:

$Y_{i}=$ es la variable dependiente (logaritmo de las ventas)

$X_{i}=$ es un vector de variables correspondientes a características de las unidades de EPS

$S_{i}=$ es la variable de asociatividad

$\varepsilon_{i}=$ es el término de error

La metodología de variables instrumentales permite corregir la correlación entre los términos $S$ y $\varepsilon$ en donde la variación en $S$ no debe estar correlacionada con el término de error. Para dicha finalidad es importante encontrar una variable $Z$ que permita satisfacer esta condición de la siguiente manera:

Correlación con $S: \operatorname{cov}(Z, S) \neq 0$

No tener correlación con $\varepsilon: \operatorname{cov}(Z, \varepsilon)=0$

Por consiguiente, el instrumento $Z$ afecta a la variable de asociatividad, pero no debe encontrarse asociada con la variable de ventas de las unidades de EPs; esta condición se la conoce como la restricción de exclusión, la cual en una primera etapa de estimación se la expresa como:

$$
\mathrm{Si}=\gamma Z_{i}+\varnothing X_{i}+\mu_{i}(2)
$$

El impacto previsto de la asociatividad sobre las ventas refleja parte del efecto producido solo por el término $S$ e incorpora solamente la variación exógena en la variable de asociatividad. La ecuación (2) se sustituye en la ecuación (1) dada la regresión de forma reducida (Khandker et al., 2010). 
Tabla 1. Estadísticas descriptivas

\begin{tabular}{|c|c|c|c|}
\hline & TOTAL & ASOCIADOS & NO ASOCIADOS \\
\hline Agentes EPS & 375,955 & 38,493 & \\
\hline Ingresos (USD) & $12,351.11$ & $15,090.37$ & $12,005.99$ \\
\hline \multicolumn{4}{|l|}{ CARACTERÍSTICAS ADMINISTRATIVAS } \\
\hline Número de trabajadores & 1.63 & 1.81 & 1.60 \\
\hline Naturaleza jurídica (personas naturales) (\%) & 98.88 & 97.28 & 99.06 \\
\hline Registros contables (posee) (\%) & 2.67 & 4.86 & 2.02 \\
\hline MUJER GERENTE (\%) & 53.45 & 43.58 & 54.82 \\
\hline Años de funcionamiento & 5.02 & 7.88 & 4.70 \\
\hline Certificación artesanal (dueño o gerente) (\%) & 8.67 & 28.76 & 6.33 \\
\hline Tipo de local (propio) (\%) & 47.33 & 40.41 & 48.08 \\
\hline \multicolumn{4}{|l|}{ TIPO DE CLIENTE } \\
\hline Cliente a nivel local (\%) & 99.34 & 98.49 & 99.46 \\
\hline Cliente a nivel provincial (\%) & 1.82 & 4.34 & 1.48 \\
\hline Cliente a nivel nacional (\%) & 0.63 & 1.84 & 0.46 \\
\hline Cliente gobierno (\%) & 0.12 & 0.36 & 0.09 \\
\hline Cliente empresas privadas (\%) & 0.53 & 1.35 & 0.43 \\
\hline Cliente público general (\%) & 99.38 & 98.33 & 99.51 \\
\hline \multicolumn{4}{|l|}{ Necesidades de financiamiento } \\
\hline Requiere financiamiento (\%) & 55.60 & 55.33 & 55.64 \\
\hline Obtención de financiamiento (\%) & 20.98 & 24.03 & 20.63 \\
\hline Monto de financiamiento (\%) & $4,117.12$ & $6,185.02$ & $3,838.55$ \\
\hline \multicolumn{4}{|l|}{ SECTOR } \\
\hline Manufactura (\%) & 9.67 & 13.52 & 9.23 \\
\hline Comercio (\%) & 57.70 & 43.05 & 59.48 \\
\hline SERVICIOS (\%) & 32.50 & 43.34 & 31.15 \\
\hline Otros $(\%)$ & 0.13 & 0.09 & 0.13 \\
\hline \multicolumn{4}{|l|}{ GASTOS CORRIENTES } \\
\hline Gastos anuales en remuneraciones (USD) & $4,965.52$ & $5,768.41$ & $4,732.29$ \\
\hline Gastos anuales en materias primas (USD) & $4,073.20$ & $4,129.86$ & $4,055.55$ \\
\hline Gastos anuales en mercadería (USD) & $7,255.23$ & $10,095.22$ & $6,986.86$ \\
\hline
\end{tabular}

Fuente: INEC-CENEC 2010.

\section{RESULTADOS}

Los datos utilizados en esta investigación corresponden al censo económico de 2010. Dentro del censo se encuentra un total de 511.130 unidades económicas. La metodología de levantamiento de información del censo establece que se debe recabar la información de cualquier unidad económica visible. Una de las ventajas que tiene la información del censo es que se registran unidades económicas sin importar su naturaleza jurídica con lo que se facilita la identificación de las unidades de EPs. La identificación de las unidades de EPS se fundamentó en 
los siguientes parámetros: ventas anuales menores de USD 60.000, dependientes asalariados en menos de 10 trabajadores y miembros integrantes de la unidad productiva de menos de 6 trabajadores. ${ }^{3}$ Bajo esta caracterización se identificaron a 375.955 unidades EPs de las cuales el $10,23 \%$ se encuentran asociadas y el $88,52 \%$ restantes no lo están. Dentro del universo de unidades económicas del país, la EPS representa el 73,55\%.

\section{ESTADÍSTICAS DESCRIPTIVAS DE LAS UNIDADES EPS}

A nivel general, las unidades de EPS presentan características ligadas a la producción informal, en promedio las ventas anuales de estos agentes se ubican en los USD 12.351, tienen un promedio de trabajadores 1,6 y casi la totalidad tienen una naturaleza jurídica de persona natural (98,88\%). Dentro de las características administrativas, solamente el 2,67\% posee registros contables, el $8,67 \%$ tiene a su gerente con certificado artesanal y el $47,33 \%$ posee local de funcionamiento propio. Estas unidades se encuentran mayoritariamente administradas por mujeres $(53,45 \%)$ y tienen un período de funcionamiento de 5,02 años en promedio.

Casi la totalidad de los clientes de las unidades EPS son de nivel local (99,34\%). Un poco más de la mitad de agentes considera que requiere financiamiento $(55,60 \%)$, pero al momento de la entrega de información solamente el 20,98\% consiguió obtener fondos con un promedio de USD 4117,12. Aproximadamente 6 de cada 10 unidades laboran en la industria del comercio, y 3 de cada 10 ejercen sus actividades en industrias vinculadas a servicios. Solamente el 9,67\% realiza producción manufacturera. ${ }^{4}$ En promedio, los gastos anuales en remuneraciones se ubican en los USD 4965,52, en materias primas USD 4073,20 y en mercadería USD 7225,23. Eso tiene correlación directa con el tipo de actividades económicas que realizan en donde la mayoría trabaja en actividades comerciales. En conjunto, se aprecia que las unidades de EPs en el Ecuador, dada la caracterización expuesta anteriormente, se concentra en actividades de producción de bajo valor agregado (comercio), tiene aspectos relacionados a la microempresa y concentra sus gastos en la adquisición de bienes para su comercialización, así como es evidente un bajo nivel de asociatividad entre ellos ya que solamente el 10,37\% reporta este tipo de vinculación (ver Tabla 1).

Separando el análisis entre unidades asociadas y no asociadas, se evidencia más niveles de formalidad en las asociadas; eso conlleva a que a nivel descriptivo las ventas promedio de las unidades asociadas sean mayores con relación a sus pares no asociados —USD 15.090,31 versus USD 12.005,99-. Las unidades asociadas poseen un mayor número de trabajadores, marginalmente tienen una menor proporción de naturaleza jurídica de persona natural, poseen una mayor proporción de unidades con registros contables así como mayor porcentaje de dueños o gerentes con certificación empresarial $-28,76 \%$ versus $6,33 \%$ en el último aspecto en mención-. Las unidades asociadas poseen una menor proporción de gerentes o administradores mujeres $-40,41 \%$ versus $48,08 \%$ - pero mayor tiempo de funcionamiento $-7,87$ años versus 4,70 años-; los clientes de ambas caracterizaciones son casi en su totalidad locales.

En relación a necesidades de financiamiento, se aprecia un porcentaje parecido; sin embargo, las unidades que los obtuvieron tienen mayor proporción entre las asociadas -24,03\% y $20,63 \%$ respectivamente- así como mayores montos recibidos - USD 6185,02 versus USD $3838,55)$. Las EPS asociadas tienen una mayor participación en sectores de manufacturas 
Tabla 2. Prueba de medias

\begin{tabular}{|c|c|c|c|c|}
\hline & $\begin{array}{l}\text { Asociados } \\
\text { (A) }\end{array}$ & $\begin{array}{c}\text { No asociados } \\
\text { (B) }\end{array}$ & $\begin{array}{l}\text { Diferencia } \\
\text { (B)-(A) }\end{array}$ & $\begin{array}{c}\text { Significancia } \\
\quad(\text { valor } \mathrm{t})\end{array}$ \\
\hline Ingresos & $15.090,37$ & $12.005,99$ & $-3.084,38$ & $-4,395$ \\
\hline Número de trabajadores & 18.053 & 16.022 & $-0,2031$ & $-39,896$ \\
\hline Naturaleza jurídica (personas naturales) & 0,9728 & 0,9906 & 0,0179 & 31,569 \\
\hline Registros contables (posee) & 0,0486 & 0,0202 & $-0,0283$ & $-35,018$ \\
\hline Mujer gerente & 0,4358 & 0,5482 & 0,1124 & 41,983 \\
\hline Años de funcionamiento & 78.753 & 46.996 & -31.757 & $-97,843$ \\
\hline Certificación artesanal (dueño o gerente) & 0,2876 & 0,0633 & $-0,2243$ & $-152,783$ \\
\hline Tipo de local (propio) & 0,4041 & 0,4808 & 0,0767 & 28,562 \\
\hline Cliente a nivel local & 0,9849 & 0,9946 & 0,0097 & 22,629 \\
\hline Cliente a nivel provincial & 0,0434 & 0,0148 & $-0,0286$ & $-40,258$ \\
\hline Cliente a nivel nacional & 0,0184 & 0,0046 & $-0,0138$ & $-33,207$ \\
\hline Cliente gobierno & 0,0036 & 0,0009 & $-0,0027$ & $-14,859$ \\
\hline Cliente empresas privadas & 0,0135 & 0,0043 & $-0,0092$ & $-23,816$ \\
\hline Cliente público general & 0,9833 & 0,9951 & 0,0117 & 27,834 \\
\hline Requiere financiamiento & 0,5533 & 0,5564 & 0,0031 & 1,149 \\
\hline Obtención de financiamiento & 0,2403 & 0,2063 & $-0,034$ & $-15,503$ \\
\hline Monto de financiamiento & $6.185,02$ & $3.838,55$ & $-2.346,48$ & $-12,700$ \\
\hline Manufactura & 0,1352 & 0,0923 & $-0,0429$ & $-26,974$ \\
\hline Comercio & 0,4305 & 0,5948 & 0,1643 & 62,116 \\
\hline Servicios & 0,4334 & 0,3115 & $-0,1218$ & $-48,490$ \\
\hline Otros & 0,0009 & 0,0013 & 0,0004 & 2,041 \\
\hline Gastos anuales en remuneraciones & $5.768,41$ & $4.732,29$ & $-1.036,12$ & $-15,150$ \\
\hline Gastos anuales en materias primas & $4.129,86$ & $4.055,55$ & $-74,31$ & $-1,363$ \\
\hline Gastos anuales en mercadería & $10.095,22$ & $6.986,86$ & $-3.108,36$ & $-31,665$ \\
\hline
\end{tabular}

Fuente: INEC-CENEC 2010.

- $13,52 \%$ versus 9,23\% - así como una menor participación en la industria de comercio $-43.05 \%$ y $59.48 \%$ respectivamente - lo que implica una mayor producción de valor agregado por parte de las unidades que si poseen asociaciones. Los gastos anuales en remuneraciones, materias primas y mercadería son mayores para las unidades asociadas; dichas diferencias no solo son descriptivas, utilizando una prueba de medias se evidencia diferencias estadísticamente significativas en las variables mencionadas entre ambos grupos (ver Tabla 2).

Dada la metodología de estimación seleccionada, conlleva un importante ejercicio el seleccionar la variable instrumental. La asociatividad, estadísticamente, no es una variable exógena, al contrario, tiene una serie de determinantes que provocan que la unidad se encuentre asociada o no; es por ello que es necesario que las variables instrumentales tengan relación con la asociatividad. En el Ecuador, las unidades EPS en relación su nivel de asociatividad, no siguen una lógica de ventas relacionada a las zonas geográficas de mayor movimiento económico. Si 
se analizan las ventas promedio a nivel cantonal, se aprecia una uniformidad en la variable con un promedio anual de USD 12.000 .

Por otro lado, los niveles de asociatividad sí registran diferencias entre los diferentes cantones del país. Por ejemplo, las unidades EPs de los cantones de las provincias de Galápagos, Azuay, Tungurahua, Napo y Pastaza registran mayores porcentajes de unidades asociadas respecto al total cantonal, con lo que se puede concluir que la lógica de asociatividad tiene una mayor relación con la zona geográfica en donde opera la unidad que con una búsqueda de lucro de la misma. Por tal motivo, se seleccionó como variable instrumental al porcentaje cantonal de unidades EPS asociadas, ya que con la misma se cumple el supuesto de restricción de exclusión; es decir, en este caso en particular, la variable debe estar altamente relacionada con la asociatividad, pero no debe tener relación con los ingresos de las unidades EPs.

\section{RESULTADOS DE LA ESTIMACIÓN}

Las estimaciones que se presentan a continuación corresponden a regresiones MCO y su respectiva corrección con la variable instrumental. Se hicieron estimaciones con y sin variables de control para comprobar el efecto que tiene la asociatividad sobre las ventas de las unidades de EPS. La variable de interés corresponde al logaritmo de las ventas anuales, mientras que la variable explicativa de interés corresponde a una dicotómica de si la unidad se encuentra asociada. Las variables de control utilizadas corresponden a: i) número de trabajadores y años de funcionamiento de la unidad como variables continuas; y ii) las variables dicotómicas de persona natural, posesión de registros contables, gerencia femenina, cliente principal en entorno local, cliente principal el gobierno central y si pertenecen al sector de manufacturas.

Se presentan cuatro estimaciones (ver Tabla 3), las dos primeras corresponden a las estimaciones MCO, mientras que las estimaciones tres y cuatro poseen la corrección por variables instrumentales. Las impares son estimaciones sin variables independientes de control mientras que las pares sí contienen los controles. Los resultados demuestran que la asociatividad sí tiene un efecto positivo en las ventas de las unidades de EPS. En las estimaciones MCO se aprecia que las unidades asociadas registran en promedio un incremento de $18,3 \%$ y $16,7 \%$ en ventas con relación a las que no se encuentran bajo un estado de asociatividad. Las correcciones realizadas con el instrumento reflejan un incremento promedio de las ventas del 12,6\% - estimación sin variables de control-y un 10,6\%. Todos los estimadores obtenidos son estadísticamente significativos al 1\% de error, con lo que se demuestra la hipótesis planteada en esta investigación.

Dentro de los justificativos del valor superior en ventas, se puede determinar que la asociatividad permite una mejor diversificación de los mercados a los que las unidades atienden. La asociatividad, adicionalmente, permite disminuir los niveles de riesgo en el caso de la obtención de financiamiento con lo que existe una mayor probabilidad de obtener recursos para el financiamiento y ampliar la capacidad instalada de las unidades. Las estadísticas descriptivas reflejaron que las unidades asociadas poseen mayor tiempo de funcionamiento y mejores niveles de formalidad, estas características pueden repercutir en la condición de asociación con lo puede existir un efecto a largo plazo de incremento en ventas de este tipo de unidades. 
Tabla 3. Estimaciones MCO y vi

\begin{tabular}{lccccc}
\hline & $(1)$ & $(2)$ & $(3)$ & $(4)$ \\
\hline Asociados (dummy) & $0.183^{* * *}$ & $0.167^{* * *}$ & $0.126^{* * *}$ & $0.106^{* * *}$ \\
\cline { 2 - 5 } & {$[0.005]$} & {$[0.005]$} & {$[0.037]$} & {$[0.031]$} \\
\hline Con controles & No & Sí & No & Sí \\
\hline Observaciones & 371,294 & 358,282 & 371,294 & 358,282 \\
\hline
\end{tabular}

Fuente: INEC-CENEC 2010. Nota: ${ }^{*} \mathrm{p}<0,10 ;{ }^{* *} \mathrm{p}<0,05 ;{ }^{* * *} \mathrm{p}<0,01$; errores estándar en corchetes.

\section{CONCLUSIONES}

La economía popular y solidaria se presenta como una nueva estructura en la que las concepciones tradicionales de la teoría de la firma se trasladan hacia nuevas funciones de maximización en torno a un beneficio comunitario y no individual. Sin embargo, los conceptos operativos de la misma se encuentran muy ligados a la economía clásica en torno al funcionamiento de la firma, la estructura de costos y la maximización del beneficio. Los agentes de la EPs representan tres cuartas partes del sistema productivo nacional, por lo que es de vital importancia concentrar esfuerzos para fortalecer a esta importante facción del aparato productivo.

Se evidenció que la asociatividad entre los agentes de la EPs tiene impactos positivos en los ingresos de los agentes, en este caso en particular, las ventas anuales de las unidades productivas, llegando a influir en un incremento del 10\% de ventas entre los agentes que se encuentran bajo un estado de asociación a gremio. No obstante, según los datos utilizados en esta investigación, se refleja que solamente 1 de cada 10 unidades de EPs tiene vínculos asociativos; si bien la metodología utilizada y los resultados obtenidos no pueden ser catalogados como una evaluación de impacto, ya que los individuos asociados difieren completamente de los no asociados en sus características observables, se logró establecer el efecto causal que tiene la asociatividad sobre las ventas. Tomando en cuenta las bondades que tiene la asociatividad dentro de los sistemas de producción, es importante crear políticas públicas que estimulen esta clase de vínculos, considerando la naturaleza productiva y los esquemas de redistribución que caracteriza a la EPS.

Considerando que una gran proporción de las unidades de EPs se concentran en la industria del comercio, la promoción de la asociatividad debe estar enfocada hacia industrias manufactureras que permitan la creación de cadenas productivas fomentando así un esquema de desarrollo endógeno en el cual la economía ecuatoriana sea cada vez más independiente de las importaciones de materias primas y de bienes de capital.

Si bien hay industrias manufactureras como la siderurgia o la producción de químicos en las que la inversión inicial sale de los parámetros de la EPs, la misma asociatividad puede ser una de las herramientas que permita a este tipo de agentes emprender inversiones de gran magnitud que permitan complementar el consumo intermedio de los bienes que son utilizados por otros agentes económicos populares y solidarios.

Se debe estimular la formalización de las unidades de la EPS en el objetivo de profundizar los fenómenos asociativos; tal como se comprobó en esta investigación, las unidades asociadas 
tienen características más formales que las no asociadas, abriendo, de esta manera, un abanico de oportunidades tales como el acceso a financiamiento con montos superiores. Estudios futuros deben medir el impacto o la causalidad que tiene el fenómeno asociativo dentro de las oportunidades de financiamiento en las unidades de EPS.

Finalmente, es necesario otorgar a las unidades de EPs que se encuentren alejadas del esquema del microcrédito, la implantación de tamaños de planta óptimos para una producción que abarque mayores segmentos de demanda. La asociatividad puede ser la herramienta que permita el otorgamiento de préstamos altos con condiciones financieras más convenientes para los actores de la economía popular y solidaria.

\section{NOTAS}

1 Burgos y Onofa (2013) encuentran mejorías en los niveles de productividad de las mipymes asociadas en el Ecuador.

2 Estudios relacionados se encuentran en Alburqueque y Valdiviezo (2008), en donde se demuestran efectos positivos en organizaciones lideradas por mujeres: Carmona y Quintero (2008) en el sector textil de Colombia, Carrillo y Hualde (2002) en sectores de maquila en el estado de Tijuana en México, López Cerdan (2003) en redes empresariales en Perú, entre otros.

3 Los parámetros se encuentran en la resolución N. ${ }^{\circ}$ CIEPS-001-2015 del Comité Interinstitucional de la Economía Popular y Solidaria del 21 de diciembre de 2015. Este comité es un órgano del poder ejecutivo integrado por el Ministerio Coordinador de Desarrollo Social, el Ministerio Coordinador de Producción, Empleo y Competitividad y el Ministerio Coordinador de Política Económica.

4 El censo no reporta unidades de producción agrícola, su metodología se enfoca en la producción industrial y de servicios. Las unidades de producción agrícola se encuentran en el Censo Agropecuario del año 2000 y en las Encuestas Anuales de Superficie y Producción Agrícola (ESPAC).

\section{REFERENCIAS}

Alburqueque, H. y Valdiviezo L. (2008). Asociatividad empresarial para el fomento del empleo femenino: un análisis para la región de Piura. Informe final. Consorcio de Investigación Económica y Social, Facultad de Economía, Universidad Nacional de Piura.

Andrabi, T., Das J., Khwaja A. (2006). Students today, teachers tomorrow? Identifying constraints on the provision of education. Massachusetts, USA: Harvard University.

Angrist, J., (1990). Lifetime earnings and the Vietnam era draft lottery: Evidence from Social Security Administration records. American Economic Review, 8o(3), 313-35.

Aragón, A. y Rubio, A. (2005, mayo-agosto). Factores explicativos del éxito competitivo: el caso de las pymes del estado de Veracruz. Contaduría y Administración, 216, 35-69.

Aragón, A. y Sánchez, G. (2005). Strategic orientation, management characteristics, and performance: A study of Spanish SMEs. Journal of Small Business Management, 43(3), 287.

Blundell R. y Costa M., (2008). Alternative approaches to evaluation in empirical microeconomics. CeMMAP Working Paper 26/o8, Centre for Microdata Methods and Practice, Institute for Fiscal Studies, London.

Bravo, S., Azcárate, J., Rojas, A. y Narváez, C. (2015). Análisis comparativo de estrategias y competitividad entre mipymes asociadas y no asociadas. Desarrollo Gerencial, 7(1), 102-121. 
Burgos S., y Onofa M., (2013) Hacia un nuevo esquema de desarrollo productivo. En H. Jácome y K. King (Eds.), Estudios industriales de la micro, pequeña y mediana empresa. (pp. 126-48). Quito, Ecuador: Flacso-MIPRo.

Carranza C. (2013). Economía de la reciprocidad: Una aproximación a la economía social y solidaria desde el concepto del don. Otra Economía, 7(12), 14-25 enero junio de 2013.

Carmona, R. y Quintero J. (julio-diciembre, 2008). Competitividad y retos en la productividad del clúster textil-confección, diseño y moda en Antioquia. Revista Ciencias Estratégicas, 16(20), 247-263.

Carrillo, J. y Hualde A. (2002). La maquiladora electrónica en Tijuana: hacia un cluster fronterizo. Revista Mexicana de Sociología, 64(3), 125-171.

Colmenarez, S., y Delgado, R. (2003). Reingeniería socioeconómica y desarrollo endógeno sostenible. Un programa macroeconómico alternativo para el desarrollo. Caracas, Venezuela: Organización Profuturo-UnESCO.

Gálvez, E., Cuéllar, K., Restrepo, C., Bernal, C. y Cortés, J. (2012). Análisis estratégico para el desarrollo de la mipyme en Iberoamérica. Cali, Colombia: Universidad del Valle.

Glewwe P., Jacoby H. (1995) An economic analysis of delayed primary school enrollment in a low income country: The role of early childhood nutrition. Review of Economic Statistics, 77(1), 159-169.

Heckman J., (1997). Instrumental Variables: A study of implicit behavioral assumptions used in making program evaluations. Journal of Human Resources, 32(3), 441-62.

Imbens G., y Andrist J. (1994). Identification and estimation of local average treatment effects. Econometrica, 62(2), 263-86.

Khandker S., Koolwal G. y Samad H., (2010). Instrumental variables estimation. In: Handbook of Impact Evaluation. Quantitative Methods and Practices (pp. 103-112). World Bank

López Cerdán, C. (2003). Redes empresariales, experiencia en la región andina. Trujillo, Perú: Minka.

Moffit, R. (2008). Estimating marginal treatment effects in heterogenous populations. Economic Working Paper Archive 539. Maryland, UsA: Jonhs Hopkins University.

Ojeda, J. (2009). La cooperación empresarial como estrategia de las Pymes del sector ambiental. Estudios Gerenciales, 25(110), 39-61.

Ravallion M. y Wodon Q. (2000). Does child labor displace schooling? Evidence on behavioral responses to an enrollment subsidy. Economic Journal, 110(462), 158-175.

Salcedo Baca, S. (2005). Políticas de seguridad alimentaria en los países andinos. Santiago, Chile: Organización de las Naciones Unidas para la Alimentación.

Schejtman, A. (1986). Campesinado y Seguridad Alimentaria. Ponencia presentada en el taller de trabajo sobre Políticas y Programas para aumentar la Producción de la Agricultura Campesina en América Latina, Instituto Latinoamericano de Planificación Económica y Social (ILPES) y el Instituto Latinoamericano de Investigaciones Sociales. Santiago, Chile.

Semykina, A. y Wooldridge J. (2005). Estimating panel data models in the presence of endogeneity and selection: Theory and application. Working Paper. Michigan, USA: Michigan State University, East Lansing.

Varian H. (2002) Microeconomía intermedia. (s. c.): Prentice Hall

Wooldridge J. (2009) Regression analysis with instrumental variables. In Introduction to Modern Econometrics. Cengage. (s. d.) 


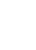

